\title{
Development of Low Cost Foot Scanner Using Foot Model
}

\author{
Ameersing Luximon ${ }^{* a}$, Zhang YiFan $^{\mathrm{a}}$, Ma Xiao $^{\mathrm{a}}$, Yan Luximon $^{\mathrm{b}}$ \\ ${ }^{a}$ Institute of Textiles and Clothing, Hong Kong Polytechnic University, Hong Kong \\ ${ }^{\mathrm{b}}$ School of Design, Hong Kong Polytechnic University, Hong Kong
}

\begin{abstract}
Three dimensional laser scanners are becoming popular for 3D shape generation. These systems are relatively expensive and hence cheaper alternatives will help manufacturers to collect sufficient data to deliver consumer goods such as footwear economically. Luximon and Goonetilleke (2004) discussed a foot shape prediction model using simple anthropometric measures. The predicted shape had a mean accuracy of $2.1 \mathrm{~mm}$ for the left foot and $2.4 \mathrm{~mm}$ for the right foot. Luximon et al. (2005) proposed two methods to generate an individual 3D foot shape from 2D information are proposed. Using the first method, they were able to predict individual shape to within a mean absolute error of $1.36 \mathrm{~mm}$ for the left foot and $1.37 \mathrm{~mm}$ for the right foot. Using the second method, the prediction was to a mean absolute error of $1.02 \mathrm{~mm}$ for the left foot and $1.02 \mathrm{~mm}$ for the right foot. This paper discusses how the similarities and differences in the foot can be used to predict foot shape and hence develop cheap foot scanner.
\end{abstract}

Keywords: Mass customization, Footwear, Foot outline, Fitting, Foot shape modeling

\section{Introduction}

Currently, ritz stick, brannock device (Brannock, 2004) and other simple measurement tools are used to capture anthropometric measures in the retail shop; however recent studies have indicated that anthropometric measures are not enough (Goonetilleke et al., 1997; Luximon, 2001). There is a need for accurate 3D foot information to enable footwear mass-customization (Luximon et al., 2003) and development of shoe-last, a 3D shape for making footwear (Adrian, 1991). Therefore, several laser scanners have been developed in different countries. Some of the commercial scanners include INfoot from Japan (cost $\approx U S \$ 34 K$ ), Yeti from Canada (cost $\approx U S \$ 90 K$ ), Scan3D from Hong Kong (cost $\approx U S \$ 46 \mathrm{~K}$ ), and Fotoscan from UK (cost $\approx \mathrm{US} \$ 25.5 \mathrm{~K}$ ). The Fotoscan does not use laser technology but instead use fringe lines and hence it is relatively cheaper. The Yeti scanner and the Scan3D have an accuracy of $0.5 \mathrm{~mm}$ or better. The Infoot scanner has an accuracy of $1 \mathrm{~mm}$. These scanner have their own advantages and disadvantages, but somehow these scanner are not cost designed for retail shop. The cost for these scanners is still expensive when we consider widespread retail application. Of course, these high end laser scanners, can be used in selected high end retail application.

In order to reduce the cost of scanning surface reconstruction model (Ding et al., 2007); anthropometric prediction model from photographic images (Hung et al., 2004); mid foot shape prediction model (Witana and Goonetilleke, 2006) have been developed. Furthermore, since anthropometric measures are widespread, girth (Zhao et al., 2008), anthropometric measures (Witana et al., 2006) and landmarks (Zhao and Goonetilleke, 2006) have been extracted from foot scanned data. The best method would be to create 3D shape using cheap widely available cameras. Then from the 3D shape, anthropometric measures and landmarks can be extracted when needed to create shoe-last and for selection of shoes, preferable in the web.

Luximon and Goonetilleke (2004) discussed a foot shape prediction model using simple anthropometric measures. The model was based on a "standard" foot and four parameters of foot length, foot width, foot height, and foot curvature. The predicted shape had a mean accuracy of 2.1 $\mathrm{mm}$ for the left foot and $2.4 \mathrm{~mm}$ for the right foot. In order to improve the technique, Luximon et al (2005) proposed two methods to generate an individual 3D foot shape from 2D information. A standard foot shape was first generated. In the first method, the foot outline and the foot height were used. Results show that each individual foot shape can be predicted within a mean absolute error of $1.36 \mathrm{~mm}$ for the left foot and $1.37 \mathrm{~mm}$ for the right foot. In the second method, the foot outline and the foot profile were used. Results show that each individual foot shape can be predicted within a mean absolute error of $1.02 \mathrm{~mm}$ for the left foot and $1.02 \mathrm{~mm}$ for the right foot. Luximon et al. (2005) have indicated that the error might be different due to image processing errors when using webcams, digital cameras or even $35 \mathrm{~mm}$ cameras. In addition, the 'standard' feet is developed using averaging method and there was more variation at the malleolus (talus) region possibly due to ankle joint movement when scanning (Luximon et al., 2005). The development of the 'standard' feet can be improved by using different averaging method and heel correction using multi-dimensional shear. 
This paper discussed the method for computation of 'standard' feet; develop several 'standard' feet based on foot type; improve the foot prediction model using multiple views; improve the foot prediction model using accurate laser scan at fixed location; and develop a prototype experimentation model to account for image processing errors. The principle of foot shape prediction and scanner development is based on similarities and differences. For similarities, we generate a standard foot shape. The standard foot shape can be average, mode or median shape. The differences are captured by low cost web cameras, anthrop meters and other simple tools.

\section{Similarities - 'standard' foot}

\subsection{Foot alignment}

Since the foot is very complex it is important to find methods to align it. Some techniques involve heel to $2^{\text {nd }}$ toe alignment; heel center line alignment; or alignment based on principle component methods. In a digital system we might look into different techniques of alignment and their advantages. However when we are developing a scanning method, it is very important to have a standardized method for foot alignment inside the scanning space to reduce acquisition errors. It should be also noted that the foot is not a fixed shape, hence deformed when we are trying to manually align the foot in a given space. Special care should be taken to avoid deformation and reduce errors.

\subsection{Ankle correction}

Based on previous studies it is found that there are larger errors at the ankle region (Luximon et al., 2005). Therefore, there is a need to find some corrective method. In this study we propose the non-linear shear method to correct the position of the heel. Using the non-linear shear, while keeping the plantar foot shape fixed the dorsal side of the foot is shifted (shear) based on a function which is dependent upon the height along the Z-axis (Fig 1.).

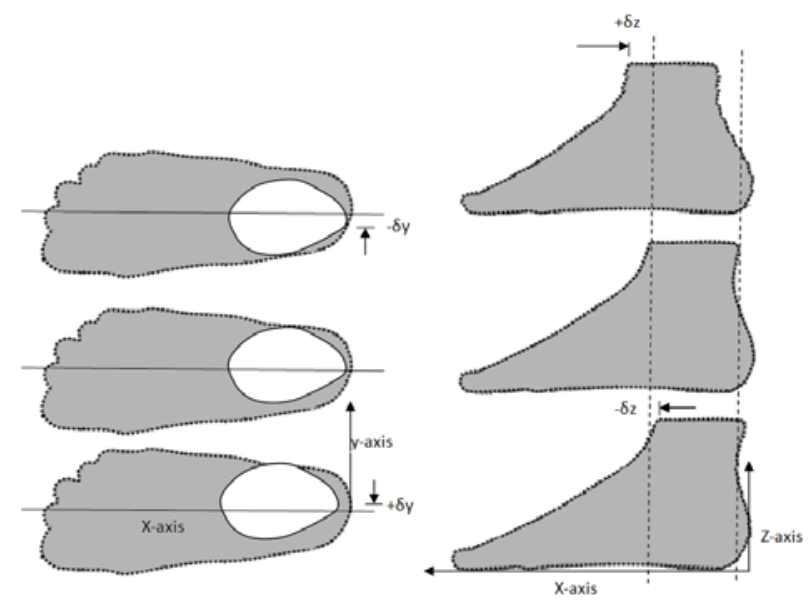

Fig. 1. Correction for ankle position.

\subsection{Computation of standard foot}

Once the foot is aligned and corrected for slight shift at the ankle joint, the foot can be classified based on toe length (length of $1^{\text {st }}$ toe > length of $2^{\text {nd }}$ toe; length of $1^{\text {st }}$ toe $=$ length of $2^{\text {nd }}$ toe, length of $1^{\text {st }}$ toe $<$ length of $2^{\text {nd }}$ toe), arch height (low arch, normal and high arch), cultures. Depending upon the size of our sample, various standard foot shapes can be generated. The method to generate standard foot may vary. It can be based on triangulation method or sectioning methods. Goonetilleke and Luximon (1999) has developed sectioning method and it seems to work well with the foot shape. The cross section at $q \%$ of foot length is given by $S_{i k}$ where $i=1, \ldots, n_{n} ; k=1, \ldots, q, . ., 99$. Alignment the sections at the centre (centroid) and using 360 points at $1^{\circ}$ interval per section, the points on cross section $S_{i 1}$ is given by ${ }_{s} p_{i k l}$ where $\left.\mathrm{i}=1, \ldots, \mathrm{n}_{\mathrm{n}} ; \mathrm{k}=1, \ldots, \mathrm{q}, \ldots, 99, \mathrm{l}=1, \ldots, 360\right)$. Standard foot can be generated using various methods. Goonetilleke and Luximon (1999) proposed average method. The points on the 'standard' foot using averaging method (shown in equation 1 ) is given by $\overline{\mathrm{p}_{\mathrm{kl}}}$ where $\mathrm{k}=1, \ldots, \mathrm{q}, . ., 99$; $\mathrm{I}=1, \ldots, 360)$.

$$
\overline{\mathrm{p}_{\mathrm{kl}}}=\frac{1}{\mathrm{n}_{\mathrm{n}}} \sum_{\mathrm{i}=1}^{\mathrm{n}_{\mathrm{n}}} \mathrm{s} \mathrm{p}_{\mathrm{ikl}}
$$


In some cases using centroid and computation of points along the curve at $1^{\circ}$ interval may not be acceptable due to huge deviation from ellipsoidal shape. Furthermore, when generating the standard foot using the average method the dorsal $\left(\mathrm{C}_{\text {Dorsal }}\right)$ and planar $\left(\mathrm{C}_{\text {plantar }}\right)$ curve are not considered. In order to improve the 'standard' foot a new method is proposed to account for dorsal $\left(\mathrm{C}_{\text {Dorsal }}\right)$ and planar $\left(\mathrm{C}_{\text {plantar}}\right)$ curve. Each cross section is separated into dorsal and plantar part. For simplicity, the dorsal curve is sectioned into equal number of points, say $n_{d}$, and the plantar curve is sectioned into equal number of points, say $n_{p}$. Assuming similar to previous case 360 points are used, then $n_{p}+n_{d}=360$. Points on the cross section $S_{i l}$ lying on the plantar side is given by ${ }_{p} p_{i k l}$ where $i=1, \ldots, n_{n} ; k=$ $\left.1, \ldots, \mathrm{q}, \ldots, 99 ; \mathrm{l}=1, \ldots, \mathrm{n}_{\mathrm{p}}\right)$. Points on the cross section $S_{\mathrm{il}}$ lying on the dorsal side is given by ${ }_{\mathrm{d}} \mathrm{p}_{\mathrm{ikl}}$ where $\mathrm{i}$ $=1, \ldots, \mathrm{n}_{\mathrm{n}} ; \mathrm{k}=1, \ldots, \mathrm{q}, . ., 99 ; \mathrm{l}=1, \ldots, \mathrm{n}_{\mathrm{d}}$ ). The points on the 'standard' foot using averaging method (shown in equation 2 ) is given by $\overline{p_{k l}}$ where $\left.k=1, . ., q, . .99 ; l=1, \ldots, 360\right)$.

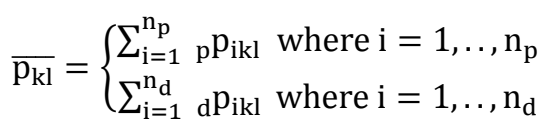

\section{Differences - Foot capture}

Once the standard foot have been generated; individual foot can be predicted based on some direct foot measurements. The direct measurement can come from anthropometers, different profiles from web cameras. Luximon and Goonetilleke (2004) used 4 anthropometric measures. More anthropometric measures can be used to reduce the prediction errors. Also principle component method can be used to select the most important anthropometric measures. In the case of Luximon et al. 2005, foot profile and outline was used. The profiles were not corrected for ankle adjustment. Ankle correction will improve the errors. In addition a mixture of anthropometric measures and profiles can be used to improve accuracy, but at the same time reducing the cost of the scanner.

\section{Scanning error}

The points on the accurate scan is ${ }_{a} p_{i}$ is $\left({ }_{a} x_{i},{ }_{a} y_{i},{ }_{a} z_{i}\right)$ where $i=1, \ldots, n_{a}$. The predicted foot shape will have points ${ }_{p} p_{k l}\left(p x_{k l}, p_{k l}, p z_{k l}\right)$ is where $k=1, \ldots, q, \ldots, 99 ; l=1, \ldots, 360$. The scanning error will be given by equation 3 and the average error given by equation 4 . It should be noted that model prediction error can be calculated, and the model prediction error will be less than scanning error. Scanning error will be slightly larger due to error in image acquisition, camera calibration and digital processing error.

$$
\begin{aligned}
& \mathrm{e}_{\mathrm{kl}}=\min \left\{\sqrt{\left({ }_{\mathrm{p}} \mathrm{x}_{\mathrm{kl}}-{ }_{\mathrm{a}} \mathrm{x}_{\mathrm{i}}\right)^{22}+\left({ }_{\mathrm{p}} \mathrm{y}_{\mathrm{kl}}-{ }_{\mathrm{a}} \mathrm{y}_{\mathrm{i}}\right)^{2}+\left({ }_{\mathrm{p}} \mathrm{z}_{\mathrm{kl}}-{ }_{\mathrm{a}} \mathrm{z}_{\mathrm{i}}\right)^{2}}\right\}, \text { where } \mathrm{i}=1, \ldots, \mathrm{n}_{\mathrm{a}} \\
& \overline{\mathrm{e}}=\frac{1}{99 * 360} \sum_{\mathrm{k}=1}^{99} \sum_{\mathrm{l}=1}^{360} \mathrm{e}_{\mathrm{kl}}
\end{aligned}
$$

\section{Conclusion}

In order to develop footwear that can match with the foot shape, three dimensional foot shape information is acquired using 3D foot scanners. Foot modelling to create low cost foot scanner is very important since there is a demand for improved footwear comfort and fit. Existing scanners are expensive and do not take advantage of the inherent similarities between the foot shapes. Also expensive laser scanner and camera system, together with linear gear to capture the foot shape is bulky and does not enable capture of dynamic foot shape. Digital image capture using web cameras, as proposed, does not require linear gear and are cheap. Data from the digital images can be processed based on multi-dimensional scaling and 'standard' foot shape. Improvement of this method using digital video and improved prediction model will be able to predict foot dynamics foot shape.

\section{Acknowledgment}

This work was supported by The Hong Kong Polytechnic University research grant (A-PH98 and $1-Z V 1 Z)$. 


\section{References}

K. C. Adrian, (1991): "American last making: procedures, scale comparisons, sizing and grading information, basic shell layout, bottom equipment standards," Arlington, MA: Shoe Trades Publishing.

Brannock, (2004): http://www.brannock.com, December 1, 2004.

Y. Ding, et al. (2007): "Partial Surface Reconstruction and Applications from Point Cloud Using RBF," Journal of Computational Information Systems, 3(6), 2479-2485.

R. S. Goonetilleke and A. Luximon, (1999): "Foot Flare and Foot Axis, " Human Factors, 41(4), 596-607.

R. S. Goonetilleke, et al., (1997): "Foot sizing beyond the 2-D Brannock method", IIE (HK). Annual Journal, 1997 - 1998, 28 - 31.

C. Y. P. Hung, et al., (2004): "Anthropometric measurements from photographic images" Proceedings of the 7th International conference on work with Computer Systems (WWCS), 29 June -2 July 2004, Kuala Lumpur, Malaysia, $764-769$.

A. Luximon, (2001): "Foot Shape Modeling for footwear fitting" PhD thesis, Hong Kong University of Science and Technology, Hong Kong.

A. Luximon and R. S. Goonetilleke, (2004): "Foot Shape Modeling". Human Factors, 46(2), $304-315$.

A. Luximon, et al. (2003): "A 3-D Methodology to Quantify Footwear Fit", In M M Tseng and F Piller (eds), The customer centric enterprise - advances in customization and personalization. (New York/Berlin: Springer) $491-499$.

A. Luximon, et al. (2005): "3D foot shape generation from 2D information" Ergonomics, 48(6), 625-641.

A. Luximon and Y. Luximon, (2009). Shoe-last Design Innovation for Better Shoe Fitting. Computers in Industry, 60(8), 621-628.

C. P. Witana and R. S. Goonetilleke, 2006, Midfoot shape when standing on soft and hard footbeds. HFES 50th Annual Meeting, October 16-20, 2006. San Francisco, California. pp. 1327-1331.

C.P. Witana, et al. (2006): Foot measurements from three-dimensional scans: A comparison and evaluation of different methods. International Journal of Industrial Ergonomics, 36(9), 789-807.

J. Zhao, et al. (2008): "Computerized girth determination for custom footwear manufacture. Computers and Industrial Engineering" 54(3). April, 359-373.

J. Zhao and R. S. Goonetilleke, (2006): "Locating anatomical points on foot from 3D point cloud data," 16th International Conference on Artificial Reality and Telexistence--Workshops (ICAT 2006) November 29- December 1st, 2006, Zhejiang University of Technology, Hangzhou, P.R.China pp. 603-607. 